\title{
COMMENTARY
}

\section{Regulatory T Cells in Idiopathic Pulmonary Fibrosis}

\section{Too Much of a Good Thing?}

\author{
Meagan W. Moore and Erica L. Herzog
}

From the Department of Internal Medicine, Section of Pulmonary, Critical Care and Sleep Medicine, Yale School of Medicine, New Haven, Connecticut

Pulmonary fibrosis is a progressive, difficult to treat, and often fatal condition characterized by the uncontrolled accumulation of extracellular matrix in the human lung. It is a feature of many disorders including environmental and occupational exposures such as asbestos and silica, genetic conditions such as surfactant and telomerase mutations, autoimmune conditions such as scleroderma and rheumatoid arthritis, therapeutic interventions such as thoracic irradiation and chemotherapies, nonimmune conditions such as uncontrolled gastroesophageal reflux disease and recurrent aspiration, as well as cryptogenic processes such as idiopathic pulmonary fibrosis (IPF). ${ }^{1}$ Although this diverse spectrum of diseases may differ in etiology, the prevailing paradigm of fibrosis suggests that the host response to prolonged or recurrent structural cell injury responses activates a pauci-immune wound healing program characterized by the accumulation of aberrantly activated, highly contractile myofibroblasts. The role of the adaptive immune system in this process, particularly the contribution of $\mathrm{CD}^{+}$ lymphocyte subsets, has been difficult to understand and highly controversial. In this issue of The American Journal of Pathology, Birjandi et $\mathrm{al}^{2}$ address the role of the regulatory subset of $\mathrm{CD}^{+} \mathrm{T}$ cells in experimentally induced pulmonary fibrosis - an area of study that has itself been laden with conflicting results.

\section{The Clinical Problem}

Although many years of animal modeling performed in various strains of lymphocyte-deficient mice demonstrate that T-cell- and B-cell-driven adaptive immune responses are dispensable for the development of experimentallyinduced lung fibrosis, ${ }^{3}$ patient care lagged behind these observations, and, for decades, first line therapy for IPF involved treatment with lymphocyte modulating agents such as corticosteroids and Azathioprine. Due in part to inability of these interventions to halt or reverse disease, combined with the failure of clinical trials aimed at suppression or modulation of classical immune responses and the emergence of fibroblast targeted therapies, ${ }^{3}$ IPF came to be viewed as a uniquely nonimmune form of pulmonary fibrosis. However, accumulating evidence now suggests that immunological mechanisms may participate in the perpetuation and maintenance of fibrotic responses in this disease. For example, circulating $\mathrm{CD}^{+} \mathrm{T}$ cells obtained from patients with IPF demonstrate autoreactive clonal expansion when stimulated with fibrotic, but not healthy, lung tissue. ${ }^{4}$ Reduced expression of the costimulatory molecule $\mathrm{CD} 28$ by circulating $\mathrm{CD}^{+}{ }^{+}$cells is predictive of poor clinical outcomes ${ }^{5}$ and a gene expression signature characterized by excessive and dysregulated $\mathrm{T}$ cell activation predict reduced event-free survival in several IPF cohorts. ${ }^{6}$ Although these findings suggest that suppression of lymphocyte responses would be beneficial in the clinical setting of IPF, the recently concluded PANTHER trial found the opposite, with IPF patients

\footnotetext{
Supported by NIH grants 1R01HL09233 and 1R01HL125850 (E.L.H.). Accepted for publication June 6, 2016.

Disclosures: E.L.H. is the PI on research grants awarded to Yale from Sanofi, Promedior, Karos, Kolltan, BiogenIdec, and Bristol Myers. E.L.H. has received consulting fees from Boehringer Ingelheim and Pfizer $(<\$ 10,000$ each) and has a provisional patent filed related to Treg targeted therapies in IPF. There is no direct real or apparent COI with the material presented herein.

Address correspondence to Erica L. Herzog, M.D., Ph.D., 300 Cedar St., TAC 441S, New Haven, CT 06520. E-mail: erica.herzog@yale.edu.
} 
randomized to receive prednisone and Azathioprine demonstrating reduced event-free survival. ${ }^{7}$ When viewed in combination, these findings suggest that skewed or aberrant adaptive immune mechanisms might amplify or orchestrate existing fibrotic response and interventions aimed at maintaining T-cell homeostasis might be of benefit in IPF.

\section{T Helper Cells in Immunopathogenesis}

The $\mathrm{T}$ helper cells, which arise from naïve $\mathrm{T}$ cells following antigen presentation and costimulation, are $\mathrm{CD}^{+}{ }^{+}$lymphocytes classified by their ability to enact effector immune responses. These include Th1 cells, which arise in response to stimulation with IL-2 or IL-12 and produce interferon (IFN)- $\gamma$ to participate in bacterial killing; Th2 cells which arise in response to IL-4 and produce a number of cytokines including IL-4 and IL-13, and are involved both in the killing of parasites and in allergic responses; and Th17 cells which are generated in part by exposure to transforming growth factor (TGF)- $\beta 1$ and IL-6 and are involved in autoimmunity through their production of IL-17 family cytokines and IL-22. Regulatory T cells (Tregs), are a CD4 ${ }^{+}$T cell population generated in response to TGF $\beta 1$ that are involved in self-tolerance and immune suppression. They are characterized by high surface expression of CD25 (the IL-2 receptor $\alpha$ chain), the transcription factor FoxP3, and the ability to control immune responses via both paracrine means involving the secretion of TGF $\beta 1$ and IL- $10^{8}$ as well as through poorly understood contact-mediated mechanisms. Tregs demonstrate the ability to suppress experimentally-induced autoimmune responses and impaired Treg function has been implicated in a variety of inflammatory and fibrosing conditions affecting humans. Experimental modeling indicates that mice with insufficient Treg quantities and function demonstrate uncontrolled immune responses and excessive mortality. ${ }^{8}$

\section{Regulatory T Cells in IPF}

Although studies in other conditions suggest that strategies aimed at reducing excessive and deleterious $\mathrm{T}$ cell responses might reduce fibrosis and remodeling, the role of Tregs in the clinical setting of IPF has been less clear. Initial reports showing reduced quantities and function of $\mathrm{CD} 4{ }^{+} \mathrm{CD} 25_{+} \mathrm{FoxP}^{+}$Tregs in the lungs and blood of patients with IPF $^{9}$ have been followed by several studies demonstrating the opposite, ${ }^{10,11}$ and, in one study, accumulation of a defective Treg population identified by the neuroimmune molecule Semaphorin 7a was associated with a more progressive clinical course. Similarly, data gleaned from animal studies show that adoptive transfer of defective, but not normal Tregs is sufficient to induce TGF $\beta 1$-driven fibrosis, ${ }^{10}$ suggesting that the function of Tregs in pulmonary fibrosis might depend on their suppressor phenotype. In another study, $\mathrm{CD} 4{ }^{+} \mathrm{CD} 25^{+}$FoxP $3{ }^{+}$Tregs showed bifunctional potential, with the ability to spontaneously cause fibroblast proliferation and fibrosis following transfer into unchallenged lungs. In contrast, deletion of FoxP3 following inhaled $\mathrm{SiO}_{2}$ led to increased accumulation of effector $\mathrm{T}$ cells and exacerbation of IL-4 mediated fibrotic responses. ${ }^{12}$ These data support the contention that Tregs also demonstrate functional heterogeneity in response to the lung microenvironment and a synthesis of the available evidence suggests that Tregs adopt differential roles in pulmonary fibrosis that depend on the combinatorial influence of intrinsic cellular properties and their response to the local milieu. Clarification of this issue is key if cell-based therapies involving Tregs are to be considered for treatment of pulmonary fibrosis.

\section{The Role of Regulatory T Cells in Experimentally-Induced Pulmonary Fibrosis}

In this issue of the $A J P$, Birjandi et $\mathrm{al}^{2}$ offer new insight into the role of regulatory $\mathrm{T}$ cells in experimentally-induced pulmonary fibrosis. The authors focused on Treg expansion as a novel potential therapy for IPF, and hypothesized that if Tregs protect against fibrosis, immunomodulatory strategies aimed at increasing the Treg population will attenuate the fibrotic response. Although this concept is not new, this study is the first to target Tregs directly in this experimental context. The authors observed that $\mathrm{CD} 4^{+} \mathrm{CD} 25^{+}{ }^{\mathrm{Foxp}} 3^{+}$Tregs accumulate in the lungs of bleomycin challenged mice. When these mice were treated with a soluble IL-2 complexed to a specific IL-2 neutralizing antibody that allows hyperstimulation of CD25 through its selective inhibition of the IL-2 receptor, detection of $\mathrm{CD}^{+}{ }^{+} \mathrm{CD} 25^{+} \mathrm{FoxP}^{+}$Tregs was further enhanced, as was collagen accumulation and histological indices of remodeling. These findings were accompanied by a hyperpolarized Th2 immune response as evidenced by reduced concentrations of the Th1 cytokine IL-18 and increased concentrations of the Th2 cytokines IL-4 and Il-13 and unaltered production of TGF- $\beta 1$. When the IL- 2 complex was delivered to mice deficient in the recombinase activating gene-2 (Rag $\left.2^{-1-}\right)$ null mice, which lack functional lymphocytes, a similar effect was not seen, indicating that the exacerbation of fibrosis caused by IL-2 complex is lymphocyte dependent. In fact, adoptive transfer of $\mathrm{CD} 25^{+} \mathrm{FoxP}^{+}$Tregs into bleomycin-exposed $\left(\mathrm{Rag}^{-/-}\right)$mice worsened mortality and fibrosis and the transferred lymphocytes were found to lose expression of FoxP3. When viewed in combination, these findings suggest that Tregs accumulate in the bleomycin-challenged lung where they ultimately lose their suppressor ability and adopt a Th2 phenotype through which they promote fibrosis and remodeling.

\section{Basic Science Insights}

As the authors suggest, ${ }^{2}$ perhaps the most significant finding of this work centers on the role of lymphocytes in pulmonary fibrosis. It has been recognized for decades that lymphocytes are not required for the development of 
bleomycin-induced pulmonary fibrosis, and the Birjandi paper $^{2}$ confirms this finding. More importantly, it shows that $\mathrm{CD}^{+}$lymphocytes in general, and Tregs in particular, are capable of exacerbating existing fibrotic response. Although various populations of $\mathrm{CD} 4^{+} \mathrm{T}$ cells have been shown to be capable of stimulating experimentally induced fibrosis, the current work ${ }^{2}$ is the first to demonstrate that adoptive transfer of any lymphocyte population is sufficient to worsen existing fibrotic responses. This finding supports the emerging paradigm that, although not required for the development of fibrosis, dysregulated $\mathrm{T}$ cell responses are sufficient to increase collagen accumulation and histological determinants of remodeling in the fibrotic mammalian lung. Further work will be required to determine the underlying mechanisms.

Another notable finding relates to lung injury. Because in this model mortality typically results from injury and precedes fibrosis, the finding that Treg transfer heightens mortality in bleomycin exposed $\mathrm{Rag} 2^{-1-}$ mice suggests that this intervention heightens the initial cell death responses that are required for and proportional to fibrosis. Such an event would be quite unexpected, since Tregs have been shown in other contexts to serve as critical regulators of epithelial injury and repair. ${ }^{13}$ Thus, it is possible that the adoptively transferred Tregs enact an excessive and accelerated repair program that simultaneously causes fibrosis in an attempt to regenerate the lung. It is also possible that the injury and inflammation caused by bleomycin overwhelm the suppressor abilities of Tregs or hyperstimulation strategies such as those used in this modeling system impede proper suppressor function. However, additional factors such as alterations in the lung microbiome, which has been recently reported to be associated with outcome in $\mathrm{IPF}^{14}$ and would be expected to influence local $\mathrm{T}$ cell responses, could account for these findings. It is also possible that the mice may simply die of overwhelming sepsis due to lack of an effective immune response caused by an excess of Tregs in the absence of effector populations. Further work is required to understand how Treg transfer heightens mortality in this experimental context.

Another notable observation centers on Treg plasticity. In this model, increased mortality was accompanied by loss of FoxP3 on transferred suppressor Tregs. This fascinating observation is consistent with earlier studies that found uncontrolled inflammation and mortality following FoxP3 deletion in otherwise healthy mice, ${ }^{8}$ and that FoxP3 deletion permits excessive effector $\mathrm{T}$ cell responses and worsened fibrosis in the silica model. ${ }^{12}$ A similar mechanism is likely at play here, as IL-2 complex therapy allows a marked Th2 polarization in treated mice, with reduction of the Th1 cytokine IL-18 and increased in Th2 cytokines such as IL-4 and IL-13. Because local and circulating levels of IL-13 are increased in many forms of lung fibrosis, and evidence of uncontrolled IL-13-driven immune responses are associated with worsened clinical outcomes, ${ }^{15}$ these data have a potential clinical correlate and might be enacted through the many mechanisms through which IL-13 is known to cause fibrosis. ${ }^{15}$ However, the source of these cytokines is unclear as the study did not determine whether the transferred Tregs themselves produced these cytokines in the IL-2 complex treated WT mice, and the cytokines were not evaluated in the $\operatorname{Rag} 2^{-1-}$ mice. Following hyperstimulation-induced loss of FoxP3, Tregs display a Th2-skewed phenotype, ${ }^{16}$ therefore, the reprogrammed Tregs themselves may produce IL13. This interpretation is further supported by the finding that adoptively transferred Tregs worsen fibrosis in $\mathrm{Rag}^{-1-}$ mice (indicating that additional lymphocyte populations are not required). It is not clear if the loss of FoxP3 is an active repolarization event or if it results from attrition caused by repetitive stimulation. It is also not clear whether this propensity is specific to Tregs or whether adoptive transfer of other $\mathrm{T}$ helper populations, or even naïve $\mathrm{T}$ cells, would have a similar effect. This question could be addressed by transfer of an irrelevant cell population as a negative control. Furthermore, it is also not clear whether all Tregs possess this capability or whether, as has been reported in other modeling systems, ${ }^{10}$ a subpopulation of abnormal Tregs accounts for this phenomenon. Clarification of this issue will be required before any conclusions can be drawn regarding whether Tregs are inherently detrimental in the setting of lung fibrosis.

Perhaps most remarkable was the finding that Treg expansion did not result in increased production of fibrosis promoting growth factors such as TGF- $\beta 1$. This observation was surprising since Tregs function in part through the secretion of TGF- $\beta 1$; prior studies have shown that Tregs promote fibrosis via TGF $\beta 1$-associated mechanisms. ${ }^{12}$ In the current study, ${ }^{2}$ because the IL-2 complex-treated lungs demonstrate a Th2 shift, it is possible that Tregs promote fibrosis by a unique TGF- $\beta 1$-independent mechanism such as that described for IL-13, ${ }^{17}$ or through the recruitment and activation of fibrosis-promoting, Th2-associated monocyte-derived cells such as fibrocytes and/or alternatively activated macrophages. However, because the authors use transcriptional evaluation rather than comparison of latent TGF- $\beta 1$ concentrations, activation efficacy, receptor expression and function, activation of canonical and noncanonical signaling pathways, and the expression and function of intrinsic inhibitors such as SMAD7, definite conclusions cannot be drawn.

\section{Translational Implications}

Although these findings are interesting and very important, care must be taken when applying them to the IPF disease state. The inhaled bleomycin model is widely used to model multiple fibrotic conditions and is not specific to IPF. In addition, because the model poorly replicates the slow development and irreversibility of IPF there is at best a modest correlation between interventions demonstrating efficacy in the bleomycin mouse model and those that are 
effective in humans. ${ }^{1}$ Thus, the failure of Treg-directed therapy to ameliorate experimentally-induced lung fibrosis may not be predictive of a similar response in humans with IPF. Better characterization of Treg heterogeneity in the lungs and blood of patients with IPF has the potential to elucidate this question. Comparison of Treg responses in patients that respond to antifibrotic therapy will be important as well, as will be the evaluation of a potential relationship with IPF-associated genes such as MUC5B, TERT, and TOLLIP. In vivo investigation of Tregs may be challenging ${ }^{1}$ without the development of models that more accurately reflect the full immune complement of the human lung; currently available humanized murine IPF models include lymphocyte deficient mice. Thus, this area may be better studied in bioengineering-based lung mimetics that allow interrogation of bidirectional crosstalk between recruited leukocytes and lung resident cell populations. ${ }^{18,19}$

\section{Conclusion and Future Directions}

The role of $\mathrm{CD}^{+} \mathrm{CD} 25^{+} \mathrm{FoxP}^{+}$Tregs in fibrosis is remarkably complex. Birjandi et $\mathrm{al}^{2}$ provide direct evidence that deregulated $\mathrm{T}$ cell responses exacerbate fibrosis in the mammalian lung through mechanisms involving loss of FoxP3 and overproduction of IL-13. The mechanisms through which these events occur and their relationship to injury will require further study, as will the applicability of these findings to human disease. Thorough evaluation of these questions will require the development of improved modeling systems that can better study the evolving and important role of Tregs in the fibrosing human lung.

\section{References}

1. Blackwell TS, Tager AM, Borok Z, Moore BB, Schwartz DA, Anstrom KJ, et al: Future directions in idiopathic pulmonary fibrosis research. An NHLBI workshop report. Am J Respir Crit Care Med 2014, 189:214-222

2. Birjandi SZ, Palchevskiy V, Xue YY, Nunez S, Kern R, Weigt SS, Lynch JP III, Chatila TA, Belperio JA: CD4 ${ }^{+} \mathrm{CD} 25^{\text {hi }}$ Foxp $3^{+}$cells exacerbate bleomycin-induced pulmonary fibrosis. Am J Pathol 2016, 186:2008-2020

3. Herzog EL, Mathur A, Tager AM, Feghali-Bostwick C, Schneider F, Varga J: Review: interstitial lung disease associated with systemic sclerosis and idiopathic pulmonary fibrosis: how similar and distinct? Arthritis Rheumatol 2014, 66:1967-1978

4. Feghali-Bostwick CA, Tsai CG, Valentine VG, Kantrow S, Stoner MW, Pilewski JM, Gadgil A, George MP, Gibson KF, Choi AM, Kaminski N, Zhang Y, Duncan SR: Cellular and humoral autoreactivity in idiopathic pulmonary fibrosis. J Immunol 2007, 179: 2592-2599

5. Gilani SR, Vuga LJ, Lindell KO, Gibson KF, Xue J, Kaminski N, Valentine VG, Lindsay EK, George MP, Steele C, Duncan SR: CD28 down-regulation on circulating CD4 T-cells is associated with poor prognoses of patients with idiopathic pulmonary fibrosis. PLoS One 2010, 5:e8959

6. Herazo-Maya JD, Noth I, Duncan SR, Kim S, Ma SF, Tseng GC, Feingold E, Juan-Guardela BM, Richards TJ, Lussier Y, Huang Y, Vij R, Lindell KO, Xue J, Gibson KF, Shapiro SD, Garcia JG, Kaminski N: Peripheral blood mononuclear cell gene expression profiles predict poor outcome in idiopathic pulmonary fibrosis. Sci Transl Med 2013, 5:205ra136

7. Idiopathic Pulmonary Fibrosis Clinical Research N, Raghu G, Anstrom KJ, King TE Jr, Lasky JA, Martinez FJ: Prednisone, azathioprine, and N-acetylcysteine for pulmonary fibrosis. N Engl J Med 2012, 366:1968-1977

8. Josefowicz SZ, Lu LF, Rudensky AY: Regulatory T cells: mechanisms of differentiation and function. Annu Rev Immunol 2012, 30:531-564

9. Kotsianidis I, Nakou E, Bouchliou I, Tzouvelekis A, Spanoudakis E, Steiropoulos P, Sotiriou I, Aidinis V, Margaritis D, Tsatalas C, Bouros D: Global impairment of CD4+CD25+FOXP3+ regulatory T cells in idiopathic pulmonary fibrosis. Am J Respir Crit Care Med 2009, 179:1121-1130

10. Reilkoff RA, Peng H, Murray LA, Peng X, Russell T, Montgomery R, Feghali-Bostwick C, Shaw A, Homer RJ, Gulati M, Mathur A, Elias JA, Herzog EL: Semaphorin 7a+ regulatory T cells are associated with progressive idiopathic pulmonary fibrosis and are implicated in transforming growth factor-beta1-induced pulmonary fibrosis. Am J Respir Crit Care Med 2013, 187:180-188

11. Galati D, De Martino M, Trotta A, Rea G, Bruzzese D, Cicchitto G, Stanziola AA, Napolitano M, Sanduzzi A, Bocchino M: Peripheral depletion of NK cells and imbalance of the Treg/Th17 axis in idiopathic pulmonary fibrosis patients. Cytokine 2014, 66:119-126

12. Lo Re S, Lecocq M, Uwambayinema F, Yakoub Y, Delos M, Demoulin JB, Lucas S, Sparwasser T, Renauld JC, Lison D, Huaux F: Platelet-derived growth factor-producing CD4+ Foxp3+ regulatory T lymphocytes promote lung fibrosis. Am J Respir Crit Care Med 2011, $184: 1270-1281$

13. Garibaldi BT, D’Alessio FR, Mock JR, Files DC, Chau E, Eto Y, Drummond MB, Aggarwal NR, Sidhaye V, King LS: Regulatory T cells reduce acute lung injury fibroproliferation by decreasing fibrocyte recruitment. Am J Respir Cell Mol Biol 2013, 48:35-43

14. Han MK, Zhou Y, Murray S, Tayob N, Noth I, Lama VN, Moore BB, White ES, Flaherty KR, Huffnagle GB, Martinez FJ; Investigators C: Lung microbiome and disease progression in idiopathic pulmonary fibrosis: an analysis of the COMET study. Lancet Respir Med 2014, 2: $548-556$

15. Homer RJ, Elias JA, Lee CG, Herzog E: Modern concepts on the role of inflammation in pulmonary fibrosis. Arch Pathol Lab Med 2011, $135: 780-788$

16. Hansmann L, Schmidl C, Kett J, Steger L, Andreesen R, Hoffmann P, Rehli M, Edinger M: Dominant Th2 differentiation of human regulatory T cells upon loss of FOXP3 expression. J Immunol 2012, 188: $1275-1282$

17. Kaviratne M, Hesse M, Leusink M, Cheever AW, Davies SJ, McKerrow JH, Wakefield LM, Letterio JJ, Wynn TA: IL-13 activates a mechanism of tissue fibrosis that is completely TGF-beta independent. J Immunol 2004, 173:4020-4029

18. Zhou Y, Peng H, Sun H, Peng X, Tang C, Gan Y, Chen X, Mathur A, Hu B, Slade MD, Montgomery RR, Shaw AC, Homer RJ, White ES, Lee CM, Moore MW, Gulati M, Geun Lee C, Elias JA, Herzog EL: Chitinase 3-like 1 suppresses injury and promotes fibroproliferative responses in Mammalian lung fibrosis. Sci Transl Med 2014, 6:240ra76

19. Huh D, Matthews BD, Mammoto A, Montoya-Zavala M, Hsin HY, Ingber DE: Reconstituting organ-level lung functions on a chip. Science 2010, 328:1662-1668 